\title{
Überdiagnose des Mammakarzinoms durch Screening
}

\section{Screening-Untersuchungen haben bei den meisten Ärzten und auch Pa- tienten ein durchaus positives Image; nur wenige sind sich der Tatsache be- wusst, dass mit Screening auch eine Menge Schaden anzurichten ist.}

- In der Screening-Terminologie versteht man unter dem Begriff der „Überdiagnose" den Prozentsatz von Krebsfällen, der ohne Screening während der verbliebenen Lebenszeit eines Menschen klinisch nicht apparent geworden wäre. Besonders heiß diskutiert wird der Wert eines Screening beim Mammakarzinom. Die Gründe dafür sind vielfältig und nicht zuletzt durch das Fehlen von Vergleichsgruppen bedingt, bei denen Trends in der Inzidenz, die dem Screening zuzuschreiben mit allgemeinen temporären Trends verglichen werden können.

Aufgrund ihres guten Meldesystems und einer hoch entwickelten medizinischen Versorgung kommen die besten Daten zu diesen Fragen aus skandinavischen Ländern. In Norwegen wurde ein bevölkerungsweites MammografieScreeningprogramm für Frauen zwischen 50 und 69 Jahren zwischen 1996 und 2005 stufenweise und regional gestaffelt eingeführt. Bei dieser Konstellation ergab sich die Möglichkeit, Verwaltungsbezirke, in denen das Programm eingeführt war, mit solchen zu vergleichen, die es noch nicht implementiert hatten. Um bessere Daten über mögliche zeitliche Trends zu erhalten, wurden auch die Inzidenzraten im Jahrzehnt vor Einführung des Screenings mit berücksichtigt.

Der Prozentsatz an Überdiagnosen wurde in zwei Ansätzen errechnet. Zum einen berechnete man die erwartete $\mathrm{Ab}$ nahme der Inzidenz im Anschluss an eine Beendigung des Screenings nach dem 69. Lebensjahr. Zum anderen verglich man die Inzidenz in der gegenwärtig gescreenten Gruppe mit der Inzidenz bei Frauen, die zwei und fünf Jahre älter als die gegenwärtig gescreenten waren. Auf diese Weise sollte die durchschnittliche sog „lead time“ Berücksichtigung finden. Darunter versteht man die Zeit, um welche die Diagnose mittels Screening früher gestellt wird als die klinische Diagnose.

Insgesamt wurden 39888 Frauen mit invasivem Mammakarzinom in die Studie eingeschlossen. 7793 Frauen erhielten ihre Diagnose, nachdem das Screeningprogramm begonnen hatte.

Die geschätzte Rate von Überdiagnosen, die dem Screening zugeschrieben werden musste, lag zwischen 18 und 25\% beim ersten Ansatz und 15-20\% beim zweiten Ansatz. Dies bedeutet, dass zwischen 15 und 25\% der Mamma-

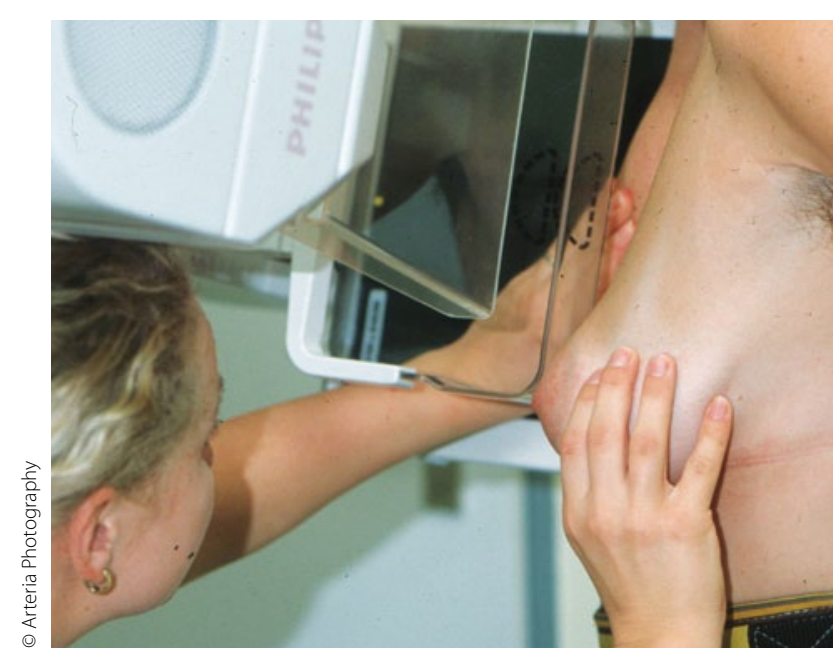

karzinome durch das Screening festgestellt wurden, bei deren Trägerinnen das Karzinom zu Lebzeiten nicht klinisch apparent geworden wäre. Die Feststellung dieser Karzinome bringt lediglich eine Vielzahl invasiver Maßnahmen und eine psychische Belastung mit sich, ohne dass sich für die Frauen daraus ein Überlebensvorteil ergibt. Pro 2500 zur Mammografie eingeladenen Frauen der genannten Altersgruppe wäre bei 6-10 Frauen mit einer Überdiagnose zu rechnen.

\section{Kommentar}

Wenn ein Screening effektiv sein soll, so müsste vor allem die Inzidenz fortgeschrittener Karzinomstadien sinken, da die Rationale hinter dem Mammografie-Screening die frühzeitige Entdeckung von Karzinomen ist, um fortgeschrittene Stadien gar nicht erst entstehen zu lassen. Genau diesen Zusammenhang zeigte eine im letzten Jahr publizierte große Populationsstudie aber nicht. In der vorliegenden Untersuchung fand man nach der Einführung des Screenings zwar einen Rückgang der Inzidenz fortgeschrittener Karzinomstadien, doch beobachtete man diesen Trend auch in den nicht gescreenten Gruppen, und zwar sowohl für Frauen in der Altersgruppe zwischen 50 und 69 Jahren als auch zwischen 70 und 79 Jahren. Somit handelt es sich wahrscheinlich nicht um einen Effekt des Mammografie-Screenings, sondern vielmehr um die Folge einer gestiegenen Aufmerksamkeit für das Problem, die möglicherweise zu einer breiteren Anwendung der Selbstuntersuchung geführt hat. Man sieht, dass es gar nicht so einfach ist, den Wert einer Screening-Maßnahme auf rationaler Basis einschätzen zu können.

H. S. FÜESSL =

\footnotetext{
- M. Kalager et al.

(Korres.: Mette Kalager, MD, Department of Epidemiology, Harvard School of Public Health, 677 Huntington Ave, Boston, MA 0215; mkalager@hsph.harvard.edu): Overdiagnosis of invasive breast cancer due to mammography screening: results from the Norwegian screening program. Ann. Int. Med. 156 (2012) 491-499
} 